\title{
Long Range Spy Robot With Night Vision
}

\author{
Pousia $\mathbf{S}^{1}$, Bharani $\mathbf{N}^{2}$, Balachandar $\mathbf{P}^{3}$, Amar V ${ }^{4}$, Kaushik $\mathbf{M}^{5}$ \\ ${ }^{1,2}$ Assistant Professor, Dept. of Electronics and Communication Engineering, Bannari Amman Institute of Technology, \\ Sathyamangalam, Tamil Nadu, India \\ ${ }^{3,4,5}$ UG Scholar, Dept. of Electronics and Communication Engineering, Bannari Amman Institute of Technology, \\ Sathyamangalam, Tamil Nadu, India
}

\begin{abstract}
The primary intention behind the undertaking is to reconnaissance on war places, data from the foes line locales; this robot has a metal locator and night vision camera to discover and observation the bombs and people in the limited zones and communicate the data to the collector material. It accommodates and saves the existence of warriors, people groups. The robot which creates for the safeguards office will help for people and save our life. Know the foes data before the adversaries get carried out. The fundamental benefit of this government operative robot is a night vision camera and metal finder. The night vision camera taps the image or recordings and sends the data to the recipient. The metal indicator identifies the bombs and metal things in underground. These robots are exceptionally valuable in these days and the future. It diminishes the work and hazard of people groups and helps them from various perspectives. These government operative robots are controlled through versatile or telephone.
\end{abstract}

Keywords: Robot, Vision camera, Metal finder, Foes line.

\section{INTRODUCTION}

Today military powers are searching for various sorts of robots for land mines, bomb identification, observation and salvage activity. Accordingly, robots decrease the danger of their setbacks and help to overcome the adversaries. Our covert operative robot depends on DTMF innovation to cover long reach. The name spy robot characterizes is impressively little it is not difficult to spy. It tends to be guided by PC or portable and can be empowered to finish assignments all alone. Our thought is to control the robot portable, the major advantage is it covers long reach and can be worked from far off territories. These days remote cameras are playing a very significant part in security issues. The camera we utilized is a remote night vision camera. It can catch picture and video data through the camera during both day and night also, send it to the beneficiary unit. Our point behind making this long-reach spy robot with a Night vision camera is chiefly to use it in war fields and spaces of mining. This framework Long Reach Spy Robot with metal and impediment recognition controls the robot's movement through call; after it recognizes metal, the bell begins and the movement of the robot is halted promptly; and in the wake of experiencing and distinguishing the snag additionally the robot, movement stops right away.

\subsection{MOTIVATION AND OBJECTIVE}

By utilizing DTMF innovation, administrators can work the robot through call regardless of reach and distance. The principle point of this robot is a closer perception of human exercises in the conflict field. It additionally has night vision cameras to send the recordings in the field which can stop the invasion of adversaries on the field. It will assist military individuals with guaranteeing wellbeing and point focuses before entering an obscure area. Also, metal finders are utilized to distinguish underground metallic items, which can shield them from obscure underground planted bombs. This framework can be controlled independently of reach and distance through call. This happens on account of the DTMF we are utilizing. By investigating all the circumstances., this framework can be utilized for close perception innovation, mining, and military application. Every one of these applications can be cultivated by introducing cameras.

\section{LITERATURE SURVEY}

This idea of a long-reach spy robot with metal and hindrance recognition is a unique framework that works independently of distance. This is because we are utilizing DTMF innovation which permits the client to work the robot using call. Here we have utilized a microcontroller ATmega328 which is the mind of the entire framework, every one of the sensors and other equipment is interfaced with this microcontroller (metal identifier, impediment locator, spy camera). To work the robot, we need to approach a portable telephone which is associated with the robot, we are utilizing two cell phones, however rather than a cell phone that is associated with robots, we can utilize GSM chips moreover. After the call is gotten (consequently), the administrator needs to send an order through the keypad of the 


\section{International Advanced Research Journal in Science, Engineering and Technology}

Vol. 8, Issue 6, June 2021

\section{DOI: $10.17148 /$ IARJSET.2021.8649}

cell phone. This framework likewise has a night vision camera, so clients can see the caught region regardless of day/night. Caught streaming can be seen on a PC. This entire framework is worked through DTMF. Priyanka Yadav1 et.al.[2] proposed a plan to plan and build up mechanical vehicles utilizing DTMF innovation for distant activity connected with a remote camera for checking purposes. While the recipient deciphers before taking care of it to a microcontroller to drive DC engines through engine driver IC for fundamental work. A metal identifier is additionally mounted on the robot body to recognize if any bomb is put underground or close by. If any metal is identified a ringer will be initiated in the control space to illuminate it. Mr. Lokesh Mehta et.al.[3] suggested that a government agent robot can likewise be constrained by a PC framework utilizing its console. Wai Mo MuKha ng et.al.[4] recommended that a covert operative robot is utilized to send video information to the intercession troop. Dheeraj Singh Patel et.al. [5], a wireless controlled robot utilizing its catches to see the live broadcast of the objective spot with a camera appended on the robot. Chiranjivi M. Deshpande et.al, [5] recommended that a telephone can be utilized as a controlling gadget to work outside gadgets utilizing DTMF innovation. So utilizing a DTMF innovation robot activity can be controlled.

\section{SPY ROBOT}

\subsection{BLOCK DIAGRAM OF PROPOSED SPY ROBOT}

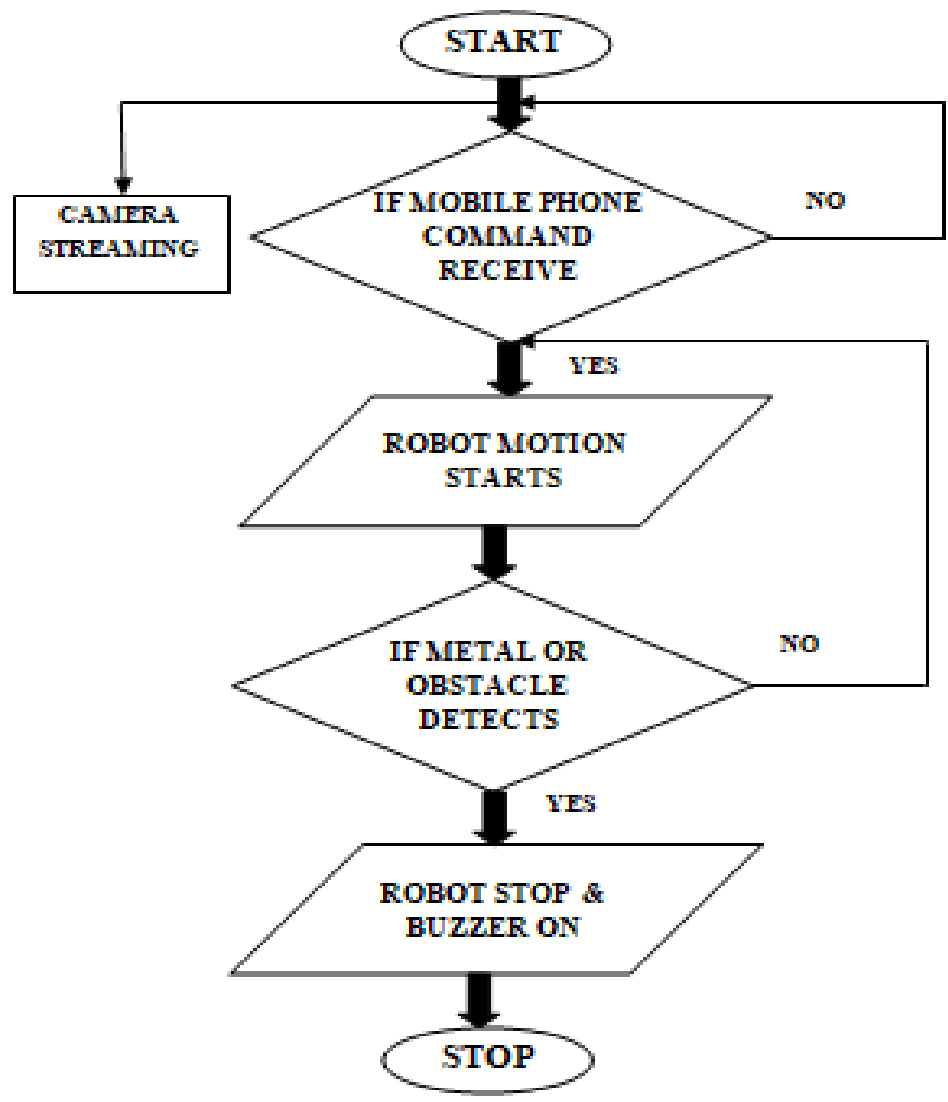

Fig.1 Block diagram of Spy Robot

3.1.1 Microcontroller: It has a place with the AVR family (ATMEGA32A). All leftover equipment peripherals are associated with the microcontroller for accepting, handling furthermore, sending information.

3.1.2 Cell Phone: Versatile is utilized to send orders to robots from anyplace on the planet. This is finished by associating any GSM cell phone to get an expansive reach to control the robot.

3.1.3 DTMF Decoder: A cell phone will be associated with the DTMF decoder for getting to the orders sent by the distant cell phone in a sound organization (DTMF) and the decoder interprets the sound organization to double identical in 4 cycles which are then shipped off the microcontroller for additional interaction.

3.1.4 Metal Identifier: A metal locator is connected to the front lower part of the robot for distinguishing the land mines and the recognized information is shipped off the microcontroller for the interaction.

3.1.5 Signal: This is the metal discovery alert as sound, during the metal recognized interaction the microcontroller stops the robot movement and conveys a message to the signal to start. Engine DRIVER: Engine drivers are associated 


\section{International Advanced Research Journal in Science, Engineering and Technology}

Vol. 8, Issue 6, June 2021

\section{DOI: $10.17148 /$ IARJSET.2021.8649}

with drive robot engines having high force prerequisites. In light of the DTMF orders, the microcontroller conveys a message to the engine driver to drive the engines for the robot's developments.

3.1.6 Engines: DC equipped engines of 12 volts appended to the robot for giving versatility to the robot. It gets a sign from the engine driver which is associated with the microcontroller.

3.1.7 Camera: Night vision remote camera chips away at the IP convention, which gives live real time video information to the distant recipient. The recipient is Cell phone or PC. In the event that the camera gets web access then live video can be seen from any place on the planet.

3.1.8 Deterrent Locator: The HC-SR04 ultrasonic sensor utilizes sonar to decide distance to an article. So it can recognize snags and keep away from deterrents.

\subsection{FLOW CHART OF PROPOSED SPY ROBOT}

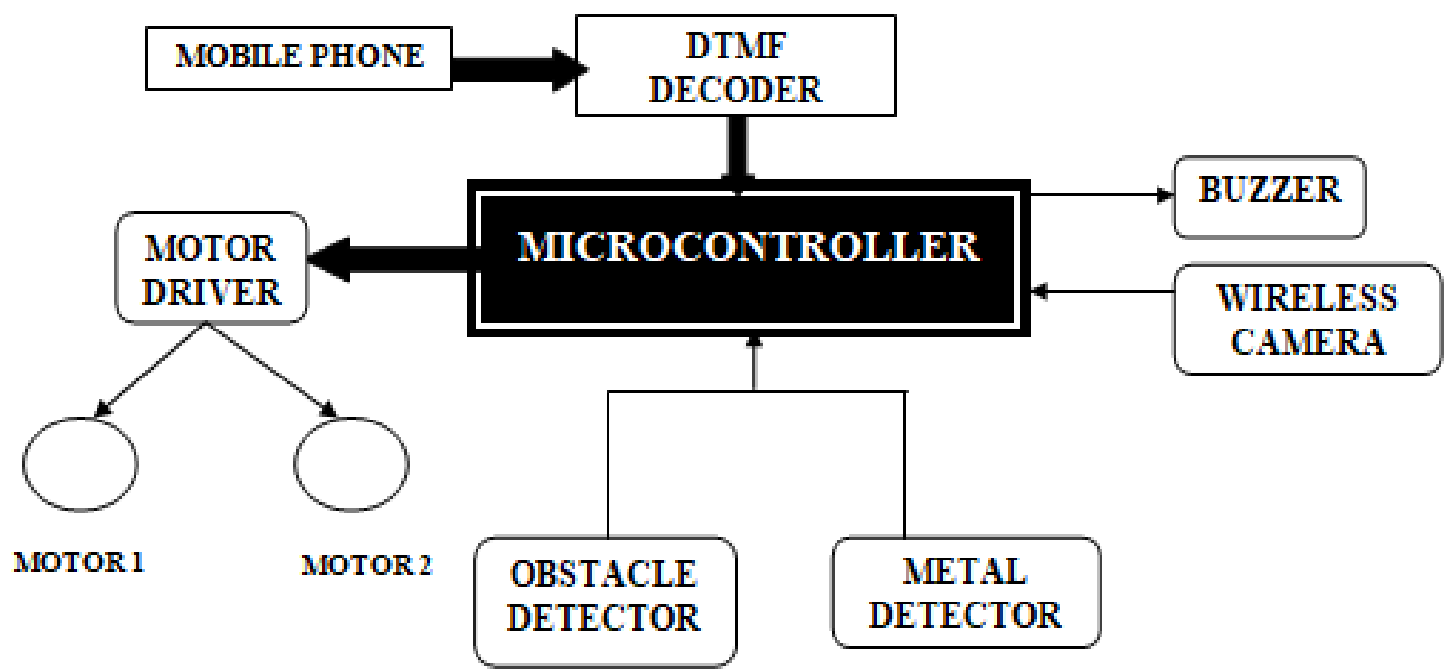

Fig.2. Flow Chart of Spy Robot

First step: Start the framework by turning on the force supply module.

Second step: The ceaseless observing of order contributions from the cell phone through DTMF decoder.

Third step: If the order is gotten, the DTMF decoder at that point begins movement of the robot depends on the order gotten. Press key 2 for Forward movement Press key 4 for Leftward movement Press key 6 for Rightward movement Press key 8 for In reverse movement Press key $*$ for halting

Fourth step: During the robot movement if metal or snag gets identified, then the robot will be halted right away furthermore, the signal beginnings giving the alarm.

\section{DESIGN METHODOLOGY}

At the underlying stage, we concocted the thought long reach spy robot with Metal and Deterrent discovery. After we contemplated the foundation work of this field. While concentrating in this field we found that there were impediments so we chose to defeat those limits however much we could. This sort of innovation is valuable in the conflict field and mining industry to supplant people where life is jeopardized. After considering our thought, we explored about new innovation in this space in our past work in college to dissect the issue. In through the keypad and DTMF decoder will add, this assists us with finding more applications utilizing this innovation. At that point we began to assemble the proposed framework and the best approach to incorporate these given frameworks to give valuable data. After this hypothetically working of this idea began which included examining the sensors to decrease the expense, block graph and stream outline. Genuine execution began in the last stage. After planning and estimations per our hypothetical conversation. In the first place, wheels are appended, to hold the whole robot.

At that point DC engines are associated with haggles Engine Driver is associated with the Arduino, so it will have high power, this arrangement is made to give our robot portability. After this DTMF Decoder is associated with the Microcontroller, here we have utilized Atmega 328 Microcontroller with the goal that all equipment associated with empower correspondence between all equipment it will deal with information sent from the two sides. A Metal is joined before the robot in the event that it recognizes metal it conveys a message to the Microcontroller and Microcontroller stops movement and conveys messages to Signal. The Signal is moreover associated with a Microcontroller. The equivalent with an Ultrasonic Locator is associated with Atmega328 Microcontroller so at whatever point any snag comes in it will stop the movement. All this activity taken by and from Microcontroller are 


\section{International Advanced Research Journal in Science, Engineering and Technology}

Vol. 8, Issue 6, June 2021

\section{DOI: $10.17148 /$ IARJSET.2021.8649}

executed in the code through Arduino UNO. After this arrangement we have utilized Cell Phone that will interface with DTMF Decoder forgetting to order send by distant portable telephone (or GSM model) interpret the organization to parallel 4 bit number which is then sent to Microcontroller for robot development. Toward the end in the wake of interfacing and association is done, at long last testing is performed and results are noted.

\section{RESULT AND DISCUSSION}

DTMF controlled robot runs by means of considering where the order is sent through the keypad of a far off cell phone. Here we are utilizing two cell phones. One is with the client and another is associated with the robot. The telephone with the client is the order transmitter unit and the second telephone on the robot is the collector unit. To start with, we need to start a call from the client cell phone to the recipient telephone, and the call is gotten consequently by means of programs answer mode work in the cell phone. At that point, the client can handle the movement of the robot by utilizing extraordinary keys on the keypad of the cell phone. At the point when any of the keys is squeezed, a specific sound is delivered from each key, this sound is caught and decoded by the DTMF decoder.

This decoded data is then sent to the microcontroller, the microcontroller measures the data and sends this handled data to engine driver IC, and afterward the engine driver IC beginnings the movement of the robot appropriately. Presently, here are the keys to control robot movement:

- When we press 6 from the client telephone, the robot will turn right.

- When we press 4 from the client telephone, the robot will turn left.

- And when we press *, the robot will stop robot movement. The sensors utilized in this framework are metal identifier and hindrance finder separately. At the point when the metal finder distinguishes the presence of metal, it sends the produced data to the microcontroller and the microcontroller conveys a high message over the port associated with the ringer and the engine, accordingly the ringer gets on and the movement of the robot stops right away.

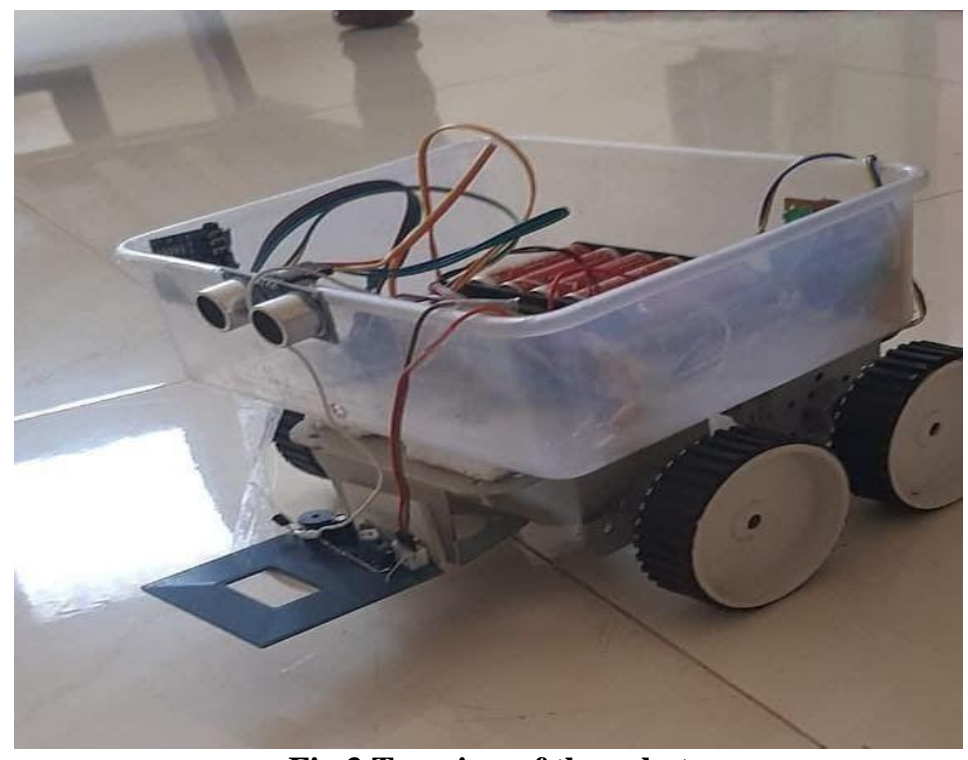

Fig.3 Top view of the robot

Fig. 3 shows the top view of the robot. The ultrasonic sensor which is utilized as a deterrent indicator ceaselessly sends ultrasonic waves, within the sight of snags the ultrasonic waves reflect back and are distinguished by the collector of the ultrasonic sensor. The time taken by the waves to reflect back subsequent to getting sent decides the distance of snags from the robot. When the presence of a snag is distinguished close to the robot, the data is shipped off the microcontroller and it will stop the robot's movement.

\section{CONCLUSION}

This paper presents another strategy for vision-based observation robot with metal identification and hindrances aversion abilities for universally useful robots in indoor environments. Experimental results in different places of deterrents and metals to check the capacity of robot to identify accuracy \& time delay. For future work, we will improve this framework for swarm mechanical technology. 


\section{International Advanced Research Journal in Science, Engineering and Technology}

Vol. 8, Issue 6, June 2021

DOI: $10.17148 /$ IARJSET.2021.8649

\section{REFERENCES}

[1]. T. S. Rappaport, —Wireless Communications: Principles and Practice,Upper Saddle River, NJ: Prentice Hall, 1996.

[2]. Dhiraj Singh Patel. Mobile operated spy robot. International Journal of Emerging Technology and Advanced Engineering (IJETAE); 2013.

[3]. Aaruni Jha, Apoorva Singh, Ravinder Turna, et al. War field spying robot with night vision camera. Journal of Network Communications and Emerging Technologies (JNCET). 2015;

[4]. Abdus Samad, Jadhav Devidas Dasharath, Dhaigude MadhukarKumar. An intelligent combat robot. International Journal of Advanced Research in Computer and Communication Engineering. 2014;

[5]. Mr. Lokesh Mehta, Mr. Pawan Sharma. Spy night vision robot withmoving wireless video camera. International Journal of ResearchEngineering Technology and Management (IJRETM); 2014

[6]. Lin, Bekey, Abney, Patrick, George, Keith (2009). "Robots in War:Issues of Risk and Ethics". Archived from the original on 2015-11-23.

[7]. Bowcott, Owen. "UK opposes international ban on developing 'killerrobots'". the Guardian. Archived from the original on 2015-0729.Retrieved 2015-07-28.

[8]. Awab Fakih, Jovita Serrao, “Cell Phone Operated Robotic Car.”International Journal of Scientific \& Engineering Research, ISSN2229-5518. 